\title{
Aortic root dilatation in young men with normally functioning bicuspid aortic valves
}

\author{
S Nistri, M D Sorbo, M Marin, M Palisi, R Scognamiglio, G Thiene
}

\begin{abstract}
Objective-To evaluate the dimensions of the aortic root in a selected population of young males with isolated normally functioning bicuspid aortic valve.

Design and setting-Echocardiographic and Doppler evaluation of conscripts with bicuspid aortic valve at the time of military pre-enrolment screening in two military hospitals.

Subjects and methods-66 consecutive young men with a normally functioning bicuspid aortic valve were studied to assess aortic size at four aortic levels: annulus, sinuses of Valsalva, supra-aortic ridge, and proximal ascending aorta; 70 consecutive normal young subjects, matched for age and body surface area, were used as controls.

Results-In men with a bicuspid aortic valve, the diameter of the aortic root was significantly larger than in controls at the sinuses $(3.16(0.37) \quad v \quad 2.87(0.31) \quad \mathrm{cm}$, $\mathrm{p}<0.001)$, at the supra-aortic ridge $(2.64$ (0.46) $v 2.47(0.28) \mathrm{cm}, \mathrm{p}=0.01)$, and at the level of the proximal ascending aorta (3.12 (0.48) v $2.69(0.28) \mathrm{cm}, \mathrm{p}<0.001)$. The prevalence of aortic root dilatation was $7.5 \%$ at the annulus $(5 / 66), 19.6 \%$ at the sinuses (13/66), $15 \%$ at the supraaortic ridge $(10 / 66)$, and $43.9 \%$ at the ascending aorta (29/66); 32 subjects (48\%) had aortic root dimensions comparable with controls, while $34(52 \%)$ had definitely abnormal aortic root dimensions. Conclusions-Aortic root enlargement in people with a bicuspid aortic valve occurs independently of haemodynamic abnormalities, age, and body size. However, there appear to be different subgroups of young adults with bicuspid aortic valves, one of which is characterised by aortic dilatation, possibly caused by a congenital abnormality of the aortic wall.

(Heart 1999;82:19-22)
\end{abstract}

Keywords: bicuspid aortic valve; aortic root dilatation

Italy

M D Sorbo

R Scognamiglio

M Marin

M Palisi

Department of Pathology, University of Padova

G Thiene

Correspondence to: Lt Col Nistri.

email: snistr@tin.it

Accepted for publication 10 February 1999 ciated with abnormalities of the aorta suc dilatation, coarctation, cystic medial necrosis, and dissection. In 1972 McKusick $^{2}$ reported the coexistence of bicuspid aortic valve and Erdheim's cystic medial necrosis, suggesting
Table 1 Baseline characteristics of control group and subjects with bicuspid aortic valve

\begin{tabular}{lll}
\hline & $\begin{array}{l}\text { Controls } \\
(n=70)\end{array}$ & $\begin{array}{l}\text { Patients } \\
(n=66)\end{array}$ \\
\hline Age (years) & $17.8(0.7)$ & $17.8(0.6)$ \\
BSA (m $\left.{ }^{2}\right)$ & $1.79(1)$ & $1.8(0.8)$ \\
LVEDVI $\left(\mathrm{ml} / \mathrm{m}^{2}\right)$ & $68.2(7)$ & $69.5(9)$ \\
Ejection fraction $(\%)$ & $59.3(8)$ & $58(7.8)$ \\
SBP $(\mathrm{mm} \mathrm{Hg})$ & $127(9)$ & $125.2(9)$ \\
DBP $(\mathrm{mm} \mathrm{Hg})$ & $77.9(5)$ & $76.4(5)$ \\
\hline
\end{tabular}

Values are mean (SD).

BSA, body surface area; DBP, diastolic blood pressure; LVEDVI, left ventricular end diastolic volume index; SBP, systolic blood pressure.

that the association was not coincidental. This observation, confirmed by other authors, ${ }^{3-6}$ has led to the hypothesis that bicuspid aortic valve and aortic abnormality may reflect a common developmental defect, namely intrinsic aortic weakness. ${ }^{7}$ Because of the influence of age, sex, and body size on aortic dimensions, we planned the present study to evaluate the dimensions of the aortic root in a selected population of young men with isolated bicuspid aortic valve and no haemodynamic disorder. Findings were compared with measurements obtained in normal subjects matched for age, sex, and body surface area.

\section{Methods}

SUBJECT SELECTION

Sixty six consecutive young men (aged 17 to 19 years, mean (SD) 17.8 (0.6) years), whose bidimensional and colour coded Doppler echocardiograms showed an isolated normally functioning bicuspid aortic valve, were studied at the Military Hospital in Padua from 1991 and in Verona from January 1993, at the time of military pre-enrolment screening. Seventy consecutive normal male subjects, matched for age, were used as controls. Both patients and controls were normotensive and their body surface areas were similar (table 1). All subjects of both groups underwent a clinical examination, a 12 lead surface ECG, and a chest $x$ ray.

\section{ECHOCARDIOGRAPHIC AND DOPPLER ANALYSIS}

Two dimensional echocardiograms were recorded with a commercially available phased array ultrasonoscope and a $2.5 \mathrm{MHz}$ or 3.5 $\mathrm{MHz}$ transducer (Hewlett Packard Sonos 1000, Andover, Massachusetts, USA). Aortic valve morphology was examined in the parasternal long and short axis views. A bicuspid aortic valve was diagnosed when only two cusps were clearly identified in systole and diastole in the short axis view (fig 1).

Aortic root dimensions were assessed at end diastole in the parasternal long axis view at four levels: the annulus, the sinuses of Valsalva, the 


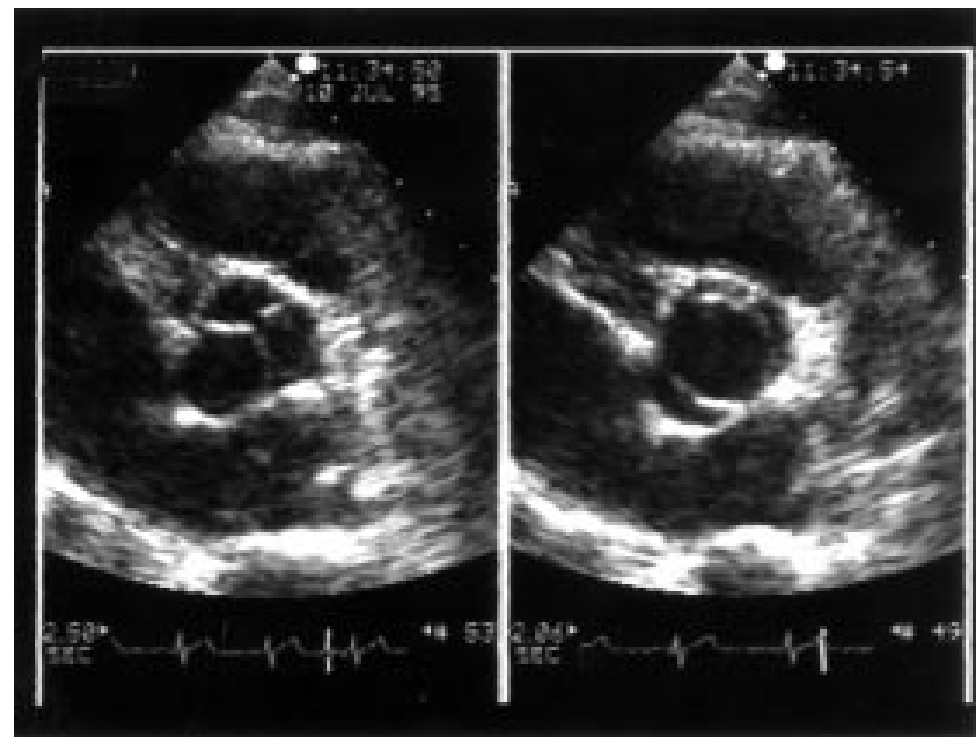

Figure 1 Echocardiographic examinations in parasternal short axis view. Left panel: diastole; the presence of a raphé at 1 o'clock simulates a normal tricuspid aortic valve. Right panel: midsystole; it is clearly demonstrated that there are only two cusps.

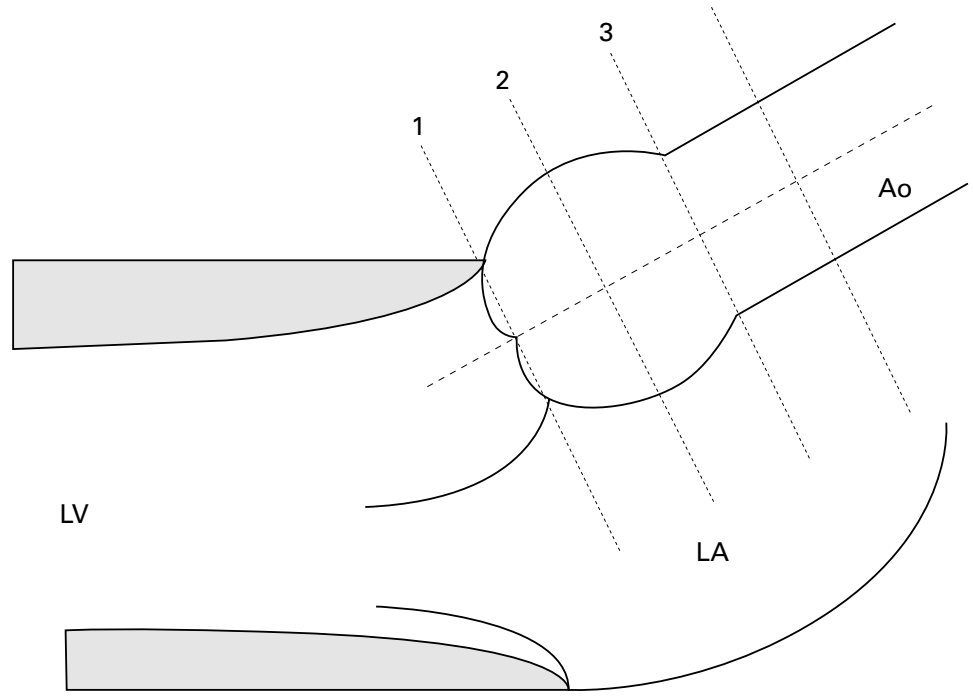

Figure 2 Diagrammatic representation of the aortic root with the sites of measurement: 1 , aortic annulus; 2 , sinuses of Valsalva; 3, supra-aortic ridge; 4, proximal ascending aorta. Ao, aorta; $L A$, left atrium; $L V$, left ventricle.

supra-aortic ridge, and the proximal ascending aorta, as previously reported. ${ }^{8} 9$ Measurements were made perpendicular to the long axis of the aorta with use of the leading edge method in views showing the largest aortic diameter (fig 2).

Aortic root measurements were made by one reader and repeated up to five times and averaged at each level. Using the parasternal approach, the aortic root could be imaged adequately and measured at each level in both

Table 2 Measurements at different levels in patients with bicuspid aortic valve and controls

\begin{tabular}{llll}
\hline & Controls $(n=70)$ & Patients $(n=66)$ & $p$ Value \\
\hline Annulus & $2.27(0.27)$ & $2.36(0.31)$ & NS \\
Sinuses of Valsalva & $2.87(0.31)$ & $3.16(0.37)$ & $<0.001$ \\
Supra-aortic ridge & $2.47(0.28)$ & $2.64(0.46)$ & 0.01 \\
Ascending aorta & $2.69(0.28)$ & $3.12(0.48)$ & $<0.001$ \\
\hline
\end{tabular}

Values are $\mathrm{cm}$ (mean (SD)). subjects and controls. The aortic arch was imaged by the suprasternal approach to rule out isthmic coarctation; in all subjects a colour Doppler guided pulsed and continuous wave study was performed to exclude isthmic coarctation.

Left ventricular volumes were calculated by an ellipsoidal biplane area-length method. The ejection fraction was calculated as [EDV $\mathrm{ESV} / \mathrm{EDV}$, where EDV is the left ventricular end diastolic volume and ESV the end systolic volume. $^{10}$

The presence of aortic regurgitation was excluded by colour Doppler in the parasternal long and short axis views, and in the apical long axis and five chamber views.

The presence of aortic valve stenosis was excluded from the apical approach, placing the sample volume of the pulsed Doppler below the valve initially for comparison with the velocities encountered at the valve level and beyond the aortic leaflets, under colour coded Doppler guidance. Peak aortic velocity was also assessed by continuous wave Doppler.

The presence and prevalence of aortic dilatation was assessed in comparison with the control group. Upper limits of aortic dimensions were considered to be the mean $+2 \mathrm{SD}$ of the values found in normal controls at each level.

\section{STATISTICS}

All data are expressed as means (SD). Difference in mean values at each aortic level between subjects and controls were analysed by Student's unpaired $t$ test. A two tailed value probability $(\mathrm{p})$ value of $<0.05$ was considered to be statistically significant.

\section{Results}

Subjects and controls were comparable with regard to left ventricular end diastolic volumes, ejection fraction, and blood pressure (table 1). No congenital heart defects other than a bicuspid aortic valve were detected in our study group. Subjects with bicuspid aortic valves had no aortic regurgitation or stenosis, and peak aortic flow velocity was comparable in subjects and controls, at $1.42(0.16) v 1.43(0.16) \mathrm{m} / \mathrm{s}$, respectively (NS).

AORTIC ROOT DIMENSIONS AND PREVALENCE OF AORTIC ROOT DILATATION

In subjects with bicuspid aortic valves the dimensions of the aortic root were larger than in the controls at each level (table 2). Differences were significant at the sinuses of Valsalva, the supra-aortic ridge, and the ascending aorta, but not at the annulus. All differences among measurements maintained their significance after indexing for body surface area.

In 32 patients (48\%), aortic root dimensions were within normal control limits at each level (group A), while in 34 (52\%) (group B) they were larger than controls - at one level in 21, and at two or more levels in 13. Subgroups were comparable with regard to blood pressure (124.7 (7.7)/76.5 (3.6) v 123.8 (13.8)/76.8 (5.3) $\mathrm{mm} \mathrm{Hg}$, respectively, NS); body surface 
Table 3 Aortic root measurements at different aortic levels in group $A$ and group $B$ patients

\begin{tabular}{llll}
\hline & Group $A(n=32)$ & Group $B(n=34)$ & p Value \\
\hline Annulus & $2.23(0.25)$ & $2.72(0.49)$ & $<0.001$ \\
Sinuses of Valsalva & $2.94(0.23)$ & $3.35(0.36)$ & $<0.001$ \\
Supra-aortic ridge & $2.38(0.26)$ & $2.87(0.46)$ & $<0.001$ \\
Ascending aorta & $2.77(0.2)$ & $3.42(0.44)$ & $<0.001$ \\
\hline
\end{tabular}

Group A, aortic root dimensions within control limits; group B, aortic root dimensions larger than control limits. Values are $\mathrm{cm}$ (mean (SD)).

area $\left(1.8(0.09) \quad v 1.78(0.1) \mathrm{m}^{2}\right.$, NS), and physical appearance (not marfanoid), but had significantly different dimensions at each aortic site (table 3). No other echocardiographic or clinical variables differed in the two subgroups.

The prevalence of aortic root dilatation, assessed in relation to the control values, was $7.5 \%$ at the annulus (5/66), $19.6 \%$ at the sinuses $(13 / 66), 15 \%$ at the sinotubular injection (10/66), and $43.9 \%$ at the ascending aorta $(29 / 66)$.

\section{Discussion}

Bicuspid aortic valve is the most common congenital heart defect. The frequent association of bicuspid aortic valve with isthmic coarctation, aortic dissection, aortic dilatation, and cystic medial necrosis has been well established..$^{3-611-14}$ The familial occurrence of aorto-cervicocephalic arterial dissection and bicuspid aortic valve has also been described. ${ }^{14}$ Aortic root dilatation, a potential clinical correlate of intrinsic medial weakness, has been investigated in patients with bicuspid aortic valve in only two previous retrospective echocardiographic studies. ${ }^{89}$ Pachulski et al reported that measurements obtained at the sinus level in 101 patients with a normally functioning or minimally stenotic bicuspid aortic valve were significantly greater than those obtained in an age and sex matched control group. ${ }^{8}$ Similarly, Hahn et $a l,{ }^{9}$ in a retrospective study of 83 patients with regurgitant, stenotic, or functionally normal bicuspid aortic valves, showed a high prevalence of aortic root enlargement in association with the bicuspid valve, irrespective of altered haemodynamics or age.

Because age, sex, and body size influence aortic dimensions, ${ }^{15} 16$ we carried out the present study on a very homogeneous population of normotensive young male subjects. Moreover, any haemodynamic perturbations that might have been caused by the bicuspid aortic valve were effectively excluded by documenting normal flow velocity with continuous wave Doppler through the aortic valve. In addition, as the subjects were referred on purely echocardiographic and Doppler criteria, any potential bias based on clinical presentation has been avoided.

Although our data confirm that there is a significant relation between bicuspid aortic valve and aortic dilatation, some details of the present study need further comment. The mean aortic dimensions of our bicuspid valve population were still within the currently accepted normal limits for age and height. ${ }^{15}$ In fact, although mean aortic size in the subjects was larger than in the normal controls, a substantial proportion of them had aortic dimensions comparable with the control group (group A). It is possible that only a subgroup of patients with bicuspid aortic valve are also affected by the congenital weakness of the aortic wall that causes premature aortic dilatation (group B), independent of any haemodynamic valvar abnormalities. However, we cannot exclude the possibility that there may be a later onset of aortic dilatation in group A subjects. The progression of aortic root dilatation in people with bicuspid aortic valve may well be variable, and not simply related to age, as has already been shown in patients with Marfan syndrome. ${ }^{17}$ The hypothesis that people with bicuspid aortic valves make up a motley population characterised by a different rate of development of aortic dilatation needs to be confirmed by a prospective study in a larger population.

In a recent population based study, ${ }^{18}$ patients with bicuspid aortic valves showed excess mortality and morbidity related to the size of the ascending aorta and the left ventricular outflow tract. Thus it is advisable to follow up patients with a bicuspid aortic valve to identify early those who are prone to aortic dilatation and potential dissection. ${ }^{19}$ Prospective studies on larger population are necessary to assess the prevalence and natural history of asymptomatic aortic dilatation in patients with bicuspid aortic valve, and to evaluate its relation to aortic dissection. Cross sectional echocardiography is the primary clinical investigation for assessing aortic size and its progression with time. ${ }^{20}$ Furthermore, by allowing full visualisation of the aortic root throughout the cardiac cycle, cross sectional echocardiography can detect the pattern of aortic root dilatation, which is a powerful prognostic marker in patients with Marfan syndrome. ${ }^{21}$ The practical value of such studies lies in confirming the need for a change in lifestyle ${ }^{22}$ and in investigating the potential role of medical treatment with $\beta$ blockers in selected patients with bicuspid aortic valve to prevent or delay the development of aortic dilatation and dissection, as demonstrated in patients with Marfan syndrome. ${ }^{23}$

\section{STUDY LIMITATIONS}

Our results suggest that there is heterogeneity of aortic dimensions in patients with bicuspid aortic valves. However, this was only an observational study at a single time point. Our findings would be greatly strengthened by a longitudinal follow up with repeated echocardiograms at regular intervals. In particular, it would be of major interest to document whether aortic root dilatation in group B patients is progressive and whether aortic diameter in group A patients remains stable over time.

A potential limitation of our study is sex bias, although a male predominance among patients with bicuspid aortic valve has been reported. However, the mean aortic dimensions and the prevalence of dilatation in our subjects were similar to those reported by others, ${ }^{89}$ and all the measurements were compared with a 
control group matched for sex, age, and body size; thus the effects of sex bias are unlikely to be important.

A local flow perturbation just above the aortic valve, caused by the abnormal opening of the bicuspid aortic valve, could induce aortic root dilatation. However, because the bicuspid valves were functioning normally and the peak aortic flow velocities in the patients and controls were comparable, it seems unlikely that flow disturbances influenced aortic size in our study population.

We did not obtain data on intraobserver and interobserver variability in the assessment of aortic dimensions in this study. However, aortic measurements were performed in strict accordance to well established echocardiographic methods, and our normal values fall within the $95 \%$ confidence interval for age and body size. ${ }^{16}$

CONCLUSIONS

Aortic root dimensions were significantly larger in young men with a normally functioning bicuspid aortic valve than in normal controls. However, some of the subjects had normal aortic dimensions in comparison with the matched controls, while others did not. Our study raises the question as to whether there are different subgroups of patients with bicuspid aortic valves, one of which is characterised by early aortic dilatation. However, the incidence and natural history of asymptomatic aortic dilatation in patients with bicuspid aortic valve are still to be determined. Our finding of heterogeneity among subjects with a bicuspid aortic valve emphasises the need for echocardiographic follow up to detect the occurrence and rate of progression of aortic dilatation.

1 Mills P, Leech G, Davies M, et al. The natural history of a non-stenotic bicuspid aortic valve. Br Heart $\mathcal{F}$ 1978;40:9517.

2 McKusick VA. Association of congenital bicuspid aortic valve and Erdheim's cystic medial necrosis. Lancet 1972; i:1026-7.
3 Becker AE, Becker MJ, Edwards JE. Anomalies associated with coarctation of the aorta. Circulation 1978;57:1067-76.

4 Edwards WD, Leaf DS, Edwards JE. Dissecting aortic aneurysm associated with congenital bicuspid aortic valve. Circulation 1978;57:1022-5.

5 Gore I. Dissecting aneurysm of the aorta in persons under forty years of age. Arch Pathol 1953;55:1-13.

6 Olson LJ, Subramanian R, Edwards WD. Surgical pathology of pure aortic insufficiency: a study of 225 cases. Mayo Clin Proc 1984;59:835-41.

7 Lindsay J. Coarctation of the aorta, bicuspid aortic valve and abnormal ascending aortic wall. Am f Cardiol 1988;61: 182-3.

8 Pachulski RT, Weinberg AL, Chan K-L. Aortic aneurysm in patients with functionally normal or minimally stenotic bicuspid aortic valve. Am 7 Cardiol 1991;67:781-2.

9 Hahn RT, Roman MT, Mogtader AH, et al. Association of aortic dilation with regurgitant, stenotic and functionally normal bicuspid aortic valves. $\mathcal{F}$ Am Coll Cardiol 1992;19: 283-8.

10 Wyatt HL, Heng MK, Meerbaum S, et al. Cross-sectional echocardiography. II. Analysis of mathematical models for quantifying volume of the formalin-fixed left ventricle. Circulation 1980;61:119-25.

11 Roberts CS, Roberts WC. Dissection of the aorta associated with congenital malformation of the aortic valve. $7 \mathrm{Am} \mathrm{Coll}$ Cardiol 1991;17:712-16.

12 Larson EW, Edwards WD. Risk factors for aortic dissection: a necropsy study of 161 cases. Am f Cardiol 1984;53:84955.

13 Basso C, Frescura C, Corrado D, et al. Congenital heart disease and sudden death in the young. Hum Pathol 1995; 26:1065-72.

14 Giusti S, Cocco P, Thiene G. Valvola aortica bicuside: una cardiopatia congenita "minore" a rischio di gravi complicanze. G Ital Cardiol 1991;21:189-201.

15 Roman MJ, Devereux RB, Kramer-Fox R, et al. Twodimensional echocardiographic aortic root dimensions in normal children and adults. Am f Cardiol 1989;64:507-12.

16 Vasan RS, Larson MG, Levy D. Determinants of echocardiographic aortic root size: the Framingham heart study. Circulation 1995;91:734-40.

17 Hwa J, Richards JG, Huang H, et al. The natural history of aortic dilatation in Marfan's syndrome. Med f Aust 1993;158:538-42.

18 Horn RA, Sarano ME, Desjardins VA, et al. The natural history of bicuspid aortic valve: a population-based study [abstract]. Circulation 1997;95:I-82A.

19 Braverman AC. Bicuspid aortic valve and associated aortic wall abnormalities. Curr Opin Cardiol 1996;11:501-3.

20 Schievink WI, Mokri B. Familial aorto-cervicocephalic arterial dissections and congenitally bicuspid aortica valve. Stroke 1995;26:1935-40.

21 Roman RJ, Rosen SE, Kramer-Fox R, et al. Prognostic significance of the pattern of aortic root dilation in the Marfan's syndrome. 7 Am Coll Cardiol 1993;22:1470-6.

22 Roberts WC. Living with a congenitally bicuspid aortic valve. Am f Cardiol 1989;64:1408-9.

23 Shores J, Berger KR, Murphy EA, et al. Progression of aortic dilatation and the benefit of long term $\beta$-adrenergic blockade in Marfan's syndrome. N Engl f Med 1994;330: 1335-41. 